\title{
Investigation on a Truncated Streamline-Traced Hypersonic Busemann Inlet
}

\author{
Yabin Xiao*, Lianjie Yue ${ }^{* *}$, Peng Gong ${ }^{*}$, and Xinyu Chang ${ }^{* * *}$ \\ Institute of Mechanics, Chinese Academy of Sciences, Beijing, China 100080
}

\begin{abstract}
A streamline-traced inlet based on the flow within the truncated Busemann inlet is designed, which has a total contraction of 7.9 and internal contraction ratio of 2.6. The boundary layer can destroy the designed flow pattern of the internal flows. Boundary layer correction that realized with the finite differential method is applied to restore the core flow to its designed pattern. CFD tools is used to investigated the flow pattern and mass flow weighted performance of the corrected inlet on its design point of Mach 5.8. Results show the reliability of the correction method and the good performance of the inlet.
\end{abstract}

\section{Nomenclature}

$\begin{array}{ll}\text { AOT } & \text { angle of truncation } \\ H & \text { enthalpy } \\ k & \text { ratio of specific heat } \\ M a & \text { Mach number } \\ M a_{\infty} & \text { free-stream Mach number } \\ P r & \text { Prandtl number } \\ P r_{t} & \text { turbulent Prandtl number } \\ r & \text { radial distance to symmetry axis } \\ V_{r} & \text { radial velocity } \\ V_{\omega} & \text { tangential velocity }\end{array}$

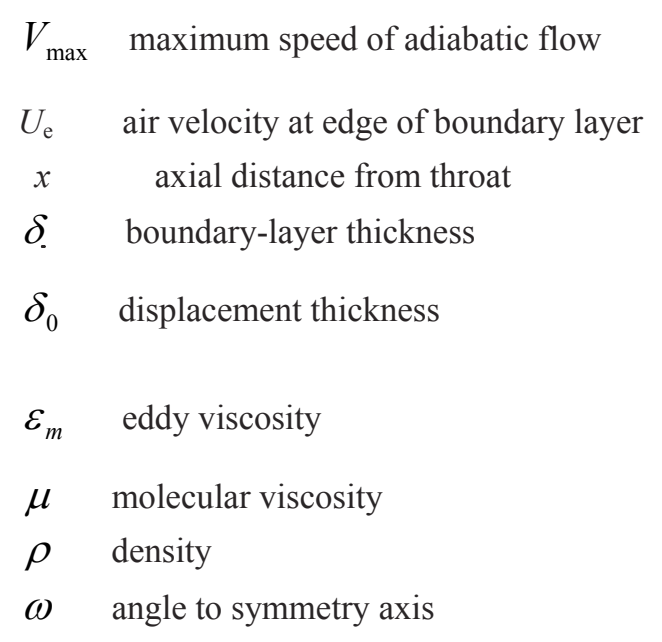

\section{Introduction}

A scramjet is a heat engine which gets energy from combustion of fuel and produces useful work to propel the aircraft 。 The inlet, combustor and nozzle are the main components of a scramjet. Incoming flow of air is captured and compressed by the inlet, high pressure and heat are added for high efficient combustion in the combustor downstream.

Design concepts for a high performance scramjet inlets are well documented in the literature, which can be characterized in terms of the planar inlets and the inward-turning inlets ${ }^{[1][2]}$ (Fig.1). In planar inlets, the flow compressions are achieved by a series of planar or quasi-planar (sidewall and 3D inlet) shock waves. Whereas, the inward turning inlets are designed to capture a portion of a known flowfield ${ }^{[3][4]}$ by specifying a leading edge and tracing the capture-perimeter streamlines through the baseline flowfield to

\footnotetext{
* $\quad$ PHD students, Key Lab of High Temperature Gas Dynamics

**Associate Professor, Key Lab of High Temperature Gas Dynamics, yuelj@imech.ac.cn,

*** Professor, Key Lab of High Temperature Gas Dynamics, Member AIAA
} 
create the inviscid waverider surfaces. Pressure rises smoothly through an infinite number of infinitesimally weak oblique shocks. Designed for full mass capture, the inward turning inlets avoid additional drag due to the spillage at design condition.

In this paper, a type of truncated streamline traced Busemann inlet is presented. The Busemann flow field within Busemann inlet is an axisymmetric internal flow defined by the Taylor-Maccoll equation:

$$
\begin{aligned}
-\frac{d^{2} V_{r}}{d \omega^{2}}\left[\frac{k+1}{2}\left(\frac{d V_{r}}{d \omega}\right)^{2}\right. & \left.-\frac{k-1}{2}\left(V_{\max }^{2}-V_{r}^{2}\right)\right]-\frac{k-1}{2}\left(\frac{d V_{r}}{d \omega}\right)^{3} \cot \omega-k V_{r}\left(\frac{d V_{r}}{d \omega}\right)^{2} \\
& +\frac{k-1}{2}\left(V_{\max }^{2}-V_{r}^{2}\right) \frac{d V_{r}}{d \omega} \cot \omega+(k-1) V_{r}\left(V_{\max }^{2}-V_{r}^{2}\right)=0
\end{aligned}
$$

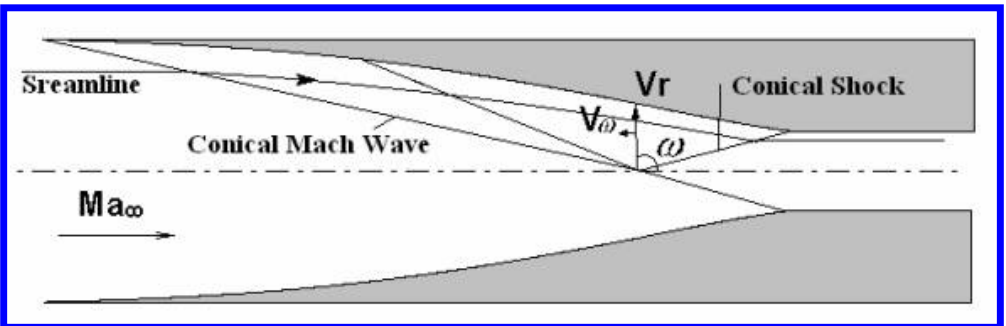

Fig 1. Full Busemann inlet

Figure 1 is a schematic illustration of the flow field. The axisymmetric internal flow consists of a set of conical Mach waves followed by a free-standing conical shock wave. All the conical Mach waves and the single freestanding conical shock wave coalesce at the apex at the origin. Under the assumption of inviscid flow, total pressure loss is considerably small.

Limited by the restriction of overall weight and size of the scramjet, it is always needed for an inlet designer to compromise between the size and the performance of an inlet. The inlet should be shortened without severe deterioration in performance. In this paper, we truncate the edge of the full Busemann inlet to obtain various baseline flow fields. Figure 2 depicts the definition of the angle of truncation (AOT). The dashed line represents the first Mach wave. The AOT is defined as the angle between the first Mach wave and the truncation line. The flow field within the full Busemann inlet of AOT of 0deg, $3 \mathrm{deg}, 4 \mathrm{deg}, 5 \mathrm{deg}$, $5.5 \mathrm{deg}$ and $6 \mathrm{deg}$ are shown in figure 3.

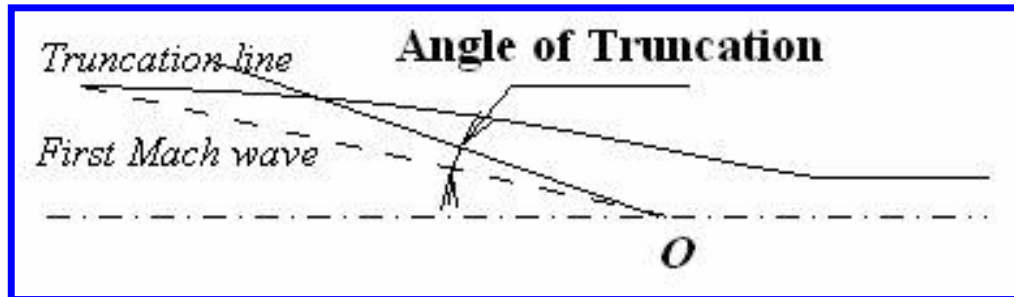

Fig 2. Definition of the angle of truncation (AOT)

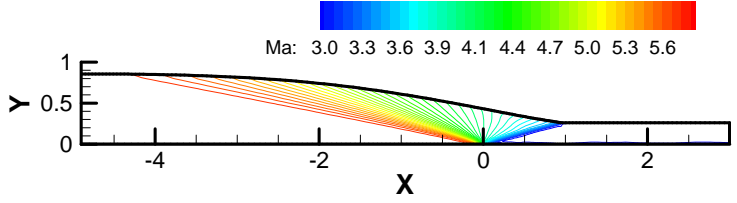

(a)AOA=0deg

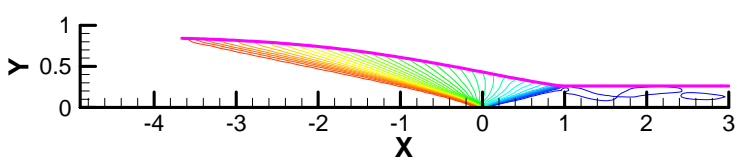

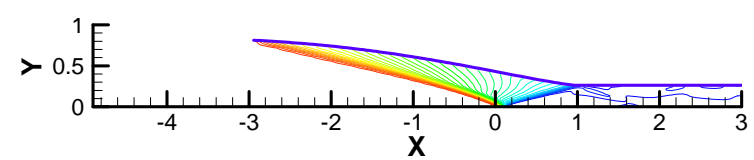

(d) $\mathrm{AOA}=5 \mathrm{deg}$

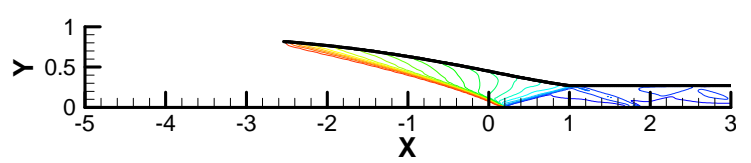


(b) $\mathrm{AOA}=3 \mathrm{deg}$

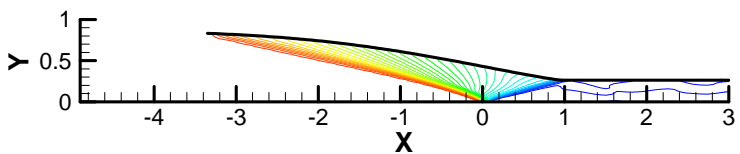

(c) $\mathrm{AOA}=4 \mathrm{deg}$ (e) $\mathrm{AOA}=5.5 \mathrm{deg}$

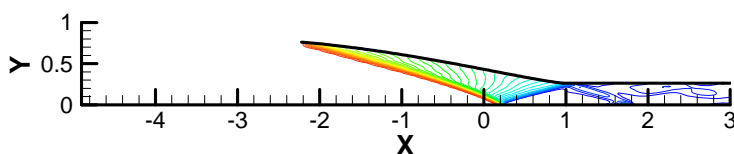

(f) $\mathrm{AOA}=6 \mathrm{deg}$

Fig 3. Flow field within a truncated full Busemann inlet

In this paper, the full Busemann inlet is designed for the case in which the Mach number is 5.8 at the entrance and 3.0 at the exit. The relative length and total pressure recovery due to the truncation are tabulated in table 1 . The best AOT is $5.5 \mathrm{deg}$, as shown in table 1 , the relative length is $2 / 3$ that of the full Busemann inlet, whereas the invscid total pressure recovery has been maintained up to $89.6 \%$.

Table 1: Relative length and total pressure recovery due to AOT

\begin{tabular}{|c|c|c|}
\hline $\begin{array}{c}\text { Angle of } \\
\text { Truncation (AOT) }\end{array}$ & $L_{t r} / L_{0}$ & $\begin{array}{c}\text { Total } \\
\text { Pressure } \\
\text { Recovery }\end{array}$ \\
\hline 0 & 1.00 & $96.4 \%$ \\
\hline 3 & 0.7905 & $93.7 \%$ \\
\hline 4 & 0.7370 & $92.4 \%$ \\
\hline 5 & 0.6895 & $90.7 \%$ \\
\hline 5.5 & 0.6674 & $89.6 \%$ \\
\hline 6 & 0.6470 & $80.6 \%$ \\
\hline
\end{tabular}

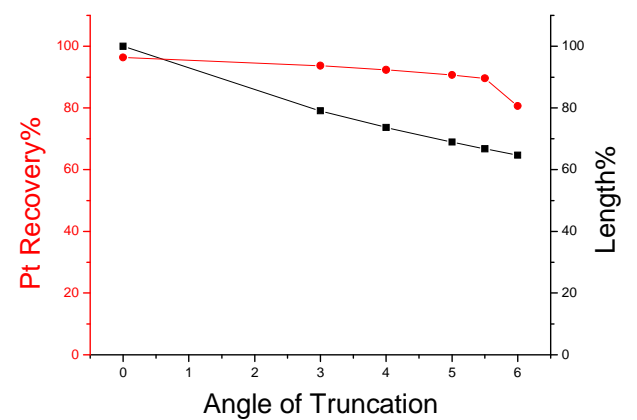

After truncation, the coalescence of conical Mach wave at the origin is not realized. There is a curved shock wave that intersects with the axis at a point that is downstream the origin ' $\mathrm{O}$ '. The reflected conical shock wave impinge at a position downstream the shoulder rather than the shoulder in the non-truncated case. However, the total pressure recovery is maintained up to $89.6 \%$. The curvature of the shock wave rises at its intersection with the axis, resulting in the high total pressure loss near the axis. As shown in the figure 4 the total pressure recovery of near the axis has come down to $63 \%$, rather than $95 \%$ that is far from the axis.

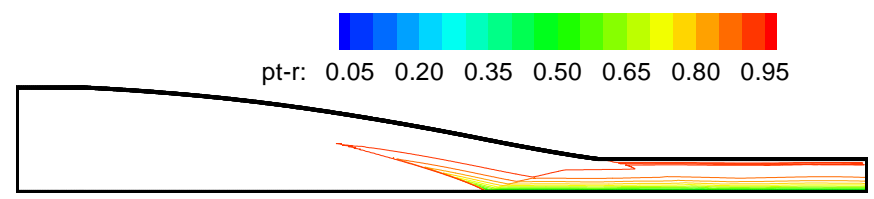

Fig 4. Total pressure recovery contours in the case of $\mathrm{AOT}=5.5 \mathrm{deg}$

\section{Design Techniques}

\section{Streamline Tracing Technique}

A supersonic inlet is said to be started when the captured flow does not choke internally and the internal flow is supersonic. The internal contraction of a fixed geometry intake is critical for self- starting performance. Streamline-tracing technique ${ }^{[3][4]}$ is introduced to reduce the internal contraction ratio to alleviate the starting problem. 
In this paper, the flow fields of full Busemann inlet and the the Busemann inlet with AOT of 5.5deg are adopted as the baseline flow fields(see figure 5). At the front view, circular and rectangular capture shapes (fig 6a and 6d) that pass through the symmetry axis of the two baseline flow fields are chosen to generate four stream-traced surfaces.

By identifying the streamlines of the baseline flowfield of full Busemann inlet which pass through the perimeter of the circular and the rectangular capture shape respectively, the stream-traced inlet with Circular Capture Shape based on the flow field within the Full Busemann Inlet (CCS-FBI, fig 6b) and Rectangular Capture Shape based on the flow field within the Full Busemann Inlet (RCS-FBI, fig 6e) are designed.

By identifying the streamlines of the baseline flowfield of the truncated Busemann inlet with AOT of 5.5deg, which pass through the perimeter of the circular and the rectangular capture shape respectively, the stream-traced inlets with Circular Capture Shape based on the flow field within the Truncated Busemann Inlet with AOT of 5.5deg(CCS-TBI-5.5, fig 6c) and Rectangular Capture Shape based on the flow field within the Truncated Busemann Inlet with AOT of $5.5 \mathrm{deg}$ (RCS- TBI- 5.5, fig 6f) are designed.

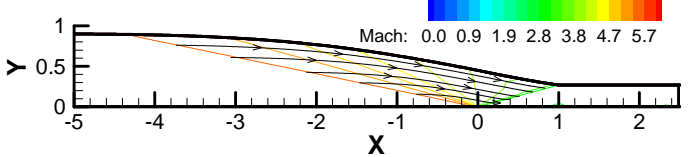

(a)Baseline flow field of full Busemann inlet

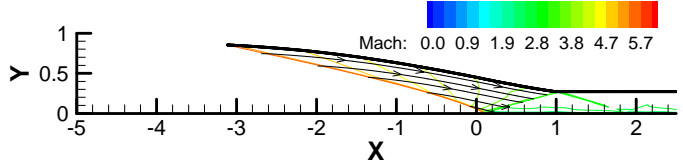

(b) Baseline flow field of truncated Busemann with AOT of 5.5deg

Fig 5. Baseline flow fields

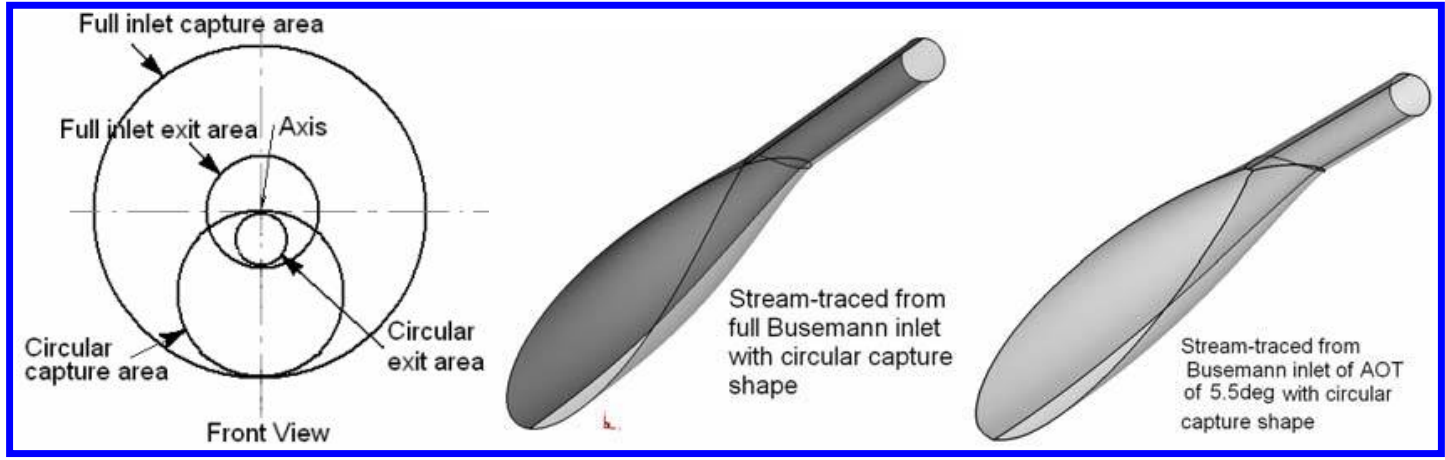

(a)

(b)CCS-FBI

(c)CCS-TBI-5.5

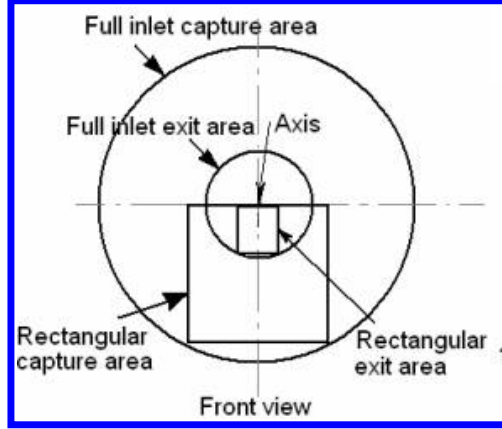

(d)

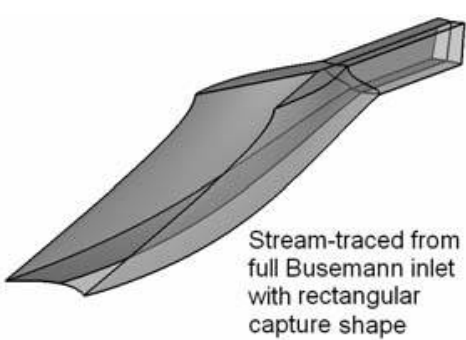

(e)RCS-FBI

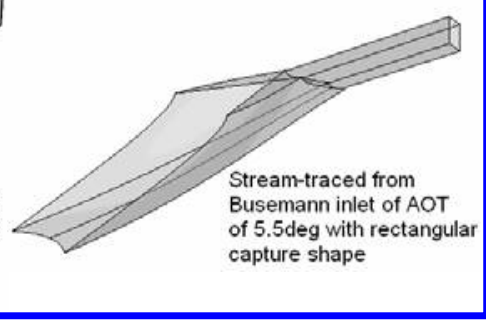

(f)RCS-TBI-5.5

Fig 6. Stream-tracing technique for designs of stream-traced inlet with circular and rectangular capture shape 


\section{Boundary Layer Correction Technique}

In reality, the viscosity effect reduces the velocity in the boundary layers on the inner surfaces of the inlet . The reduced velocity implies a decrease in the capacity of the inlet. One effect of the boundary layer is that it acts as a blockage in the cannel and actually adds to the contraction ratio which can degrade the designed internal flow field and decrease the start performance at the same time. Figure 7 depicts the effect of the boundary layer on the internal flow within the full Busemann inlet. Because of the displacement, the isentropic compression in not realized, the internal conical flow has been converted into a totally different pattern, and the total pressure recovery of the core flow decreases to $60 \%$ at the exit.

For the stream-traced inlets, the displacement of the boundary layer and the shock wave boundary layer interaction result in an additional contraction, which will cause the steady unstart. The flow pattern within CCS-TBI-5.5(circular capture shape based on the flow field within the truancated Busemann inlet with AOT of $5.5 \mathrm{deg}$ ) was numerical simulated. Figure 8 depicts the shock wave structure of the uncorrected CCS-TBI-5.5, where there is a normal shock wave stand in front of the cowl lip. The inlet is unstarted. An unstarted intake suffers a significant loss of mass capture and total pressure. It is necessary to conduct the boundary layer correction.

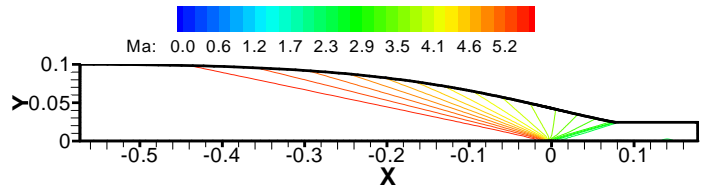

(a) Mach contours of inviscid full Busemann inlet

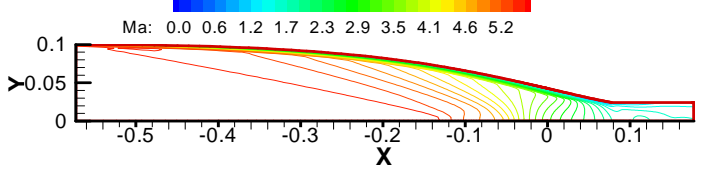

(c) Mach contours of viscid full Busemann inlet uncorrected

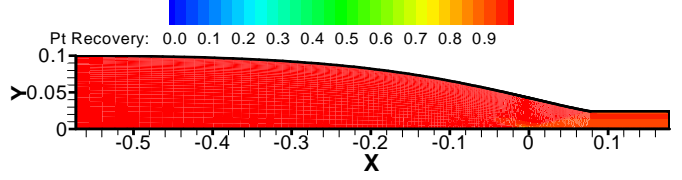

(b) Total pressure recovery contours of inviscid full Busemann inlet

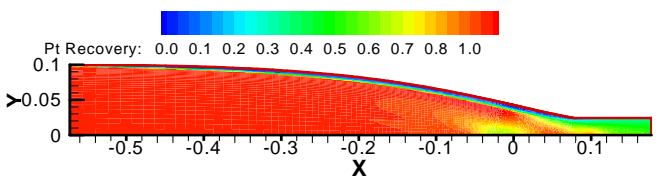

(d)Total pressure recovery contours of viscid full Busemann inlet

Fig 7. Viscosity effect on the internal flow

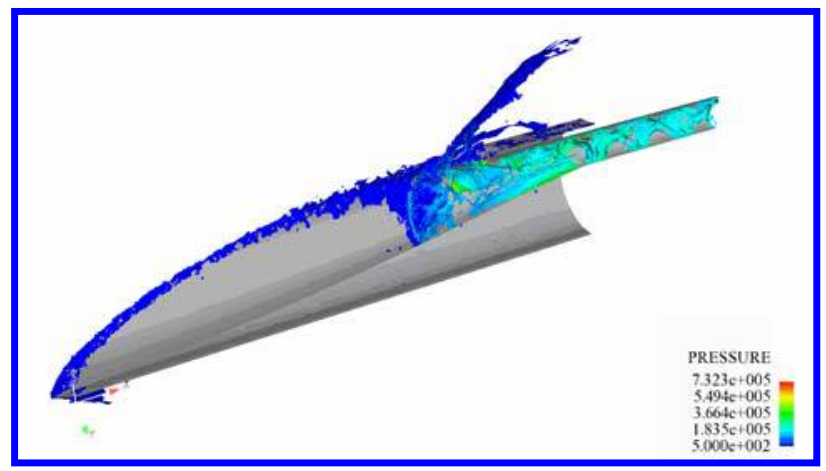

Fig 8. Shock wave structure of the unstarted CCS-TBI-5.5

A correction can be applied to the internal diameter of the inlet by increasing the axial distance with 
the displacement thickness of the boundary layer to compensate for the blockage of the boundary layers ${ }^{[5]}$, as shown in fig 9a. The displacement thickness can be calculated by numerically solving the Reynolds averaged turbulent compressible boundary layer equations with pressure gradient (2.1),(2.2),(2.3) with the finite differential method presented by Cebeci and Bradshaw ${ }^{[6]}$. For stream-traced inlets, normal vector of the surfaces are calculated, along which the surfaces are moved the distance of local displacement thickenss. Figure $9 \mathrm{~b}$ illustrates the normal vectors colorized by the local displacement thickness. Figure 10 illustrates the corrected stream-traced inlets. Displacement thickness of the boundary layer on the lower side of inlets is larger, so the correction amount is larger.

$$
\begin{aligned}
& \frac{\partial \rho u}{\partial x}+\frac{\partial}{\partial y} \overline{\rho u}=0 \\
& \rho u \partial \frac{\partial u}{\partial x}+\overline{\rho v} \frac{\partial u}{\partial x}=-\rho_{e} u_{e} \frac{d u_{e}}{d x}+\frac{\partial}{\partial y}\left(\left(\mu+\rho \varepsilon_{m}\right) \frac{\partial u}{\partial y}\right) \\
& \rho u \frac{\partial H}{\partial x}+\overline{\rho u} \frac{\partial H}{\partial y}=\frac{\partial}{\partial y}\left\{\left(\frac{\mu}{\operatorname{Pr}}+\rho \frac{\varepsilon_{m}}{\operatorname{Pr}_{t}}\right) \frac{\partial H}{\partial y}+\left[\mu\left(1-\frac{1}{\operatorname{Pr}}\right)+\rho \varepsilon_{m}\left(1-\frac{1}{\operatorname{Pr}_{t}}\right)\right] u \frac{\partial u}{\partial y}\right\} \\
& \delta^{*}=\int_{0}^{\delta}\left(1-\frac{\rho u}{\rho_{\mathrm{e}} u_{e}}\right) d h
\end{aligned}
$$

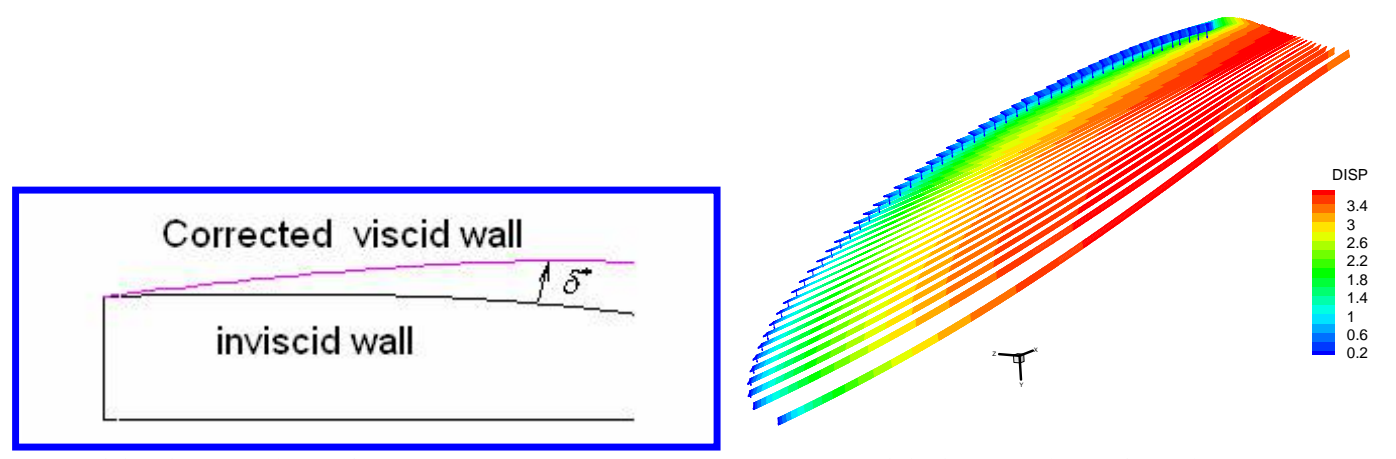

(a) Correction of 2D

(b) Correction of 3D inlet

axisymmetric inlet

local normal vector and displacement thickness

Fig 9. Correction of $2 D$ and $3 D$ inlets

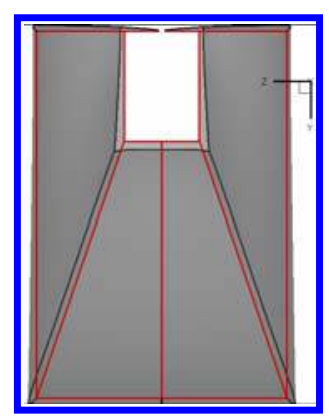

(a) Front view of corrected(black edge) and uncorrected(red edge) RCS-FBI

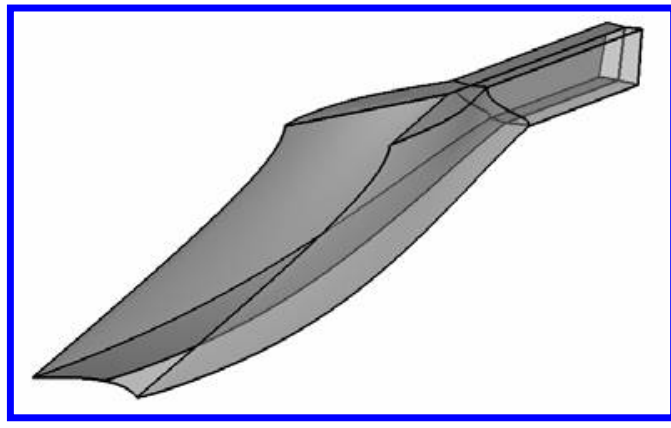

(b) Angular view of corrected RCS-FBI 


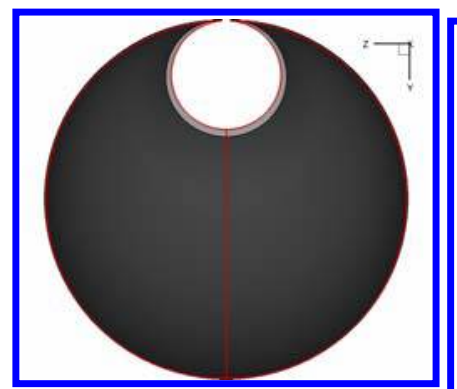

(c) Front view of

corrected(black edge) and

uncorrected(red edge) CCS-FBI

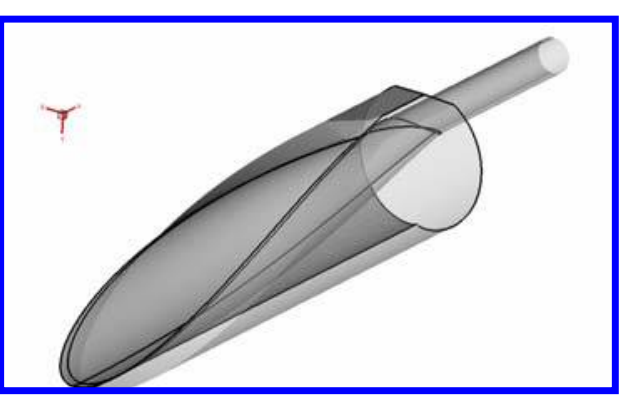

(b) Angular view of corrected

CCS-FBI

Fig 10. Comparison of corrected and uncorrected inlets

\section{Analysis}

This paper describes a numerical effort to explore the flow pattern within the stream-traced inlet based on the baseline flow field of the truncated Busemann inlet. A number of hypersonic flow examples including the two dimensional axisymmetric Busemann inlet, the stream-traced inlets based on the truncated Busemann flow field are described in this paper. The assumption of full turbulence from the leading edge of the inlet is adopted not without justification yet. All computations were done using Fluent 6.3 with $k-\omega \mathrm{SST}$ two-equation eddy viscosity model without body forces or external heat addition. Fluent is a commercial Navier-Stokes solver for either compressible or incompressible fluid flows. It features a second order upwind discretisation scheme and the AUSM flux type.

For three dimensional cases, the computational domain covers the entire internal flow and a part of the external flow of the inlet model, as shown in figure 11a. Figure $11 \mathrm{~b}$ and $11 \mathrm{c}$ show the computational grid. A hybrid unstructured grid is employed that is comprised by the tetrahedrons in the core flow region and the 24 layers of prisms near the wall. The computations were carried out on a 420,000 cell grid. At least three cells inside the viscous sublayer and $\mathrm{y}^{+} \leq 0.89$ at the first layer of grid centroids away from the wall.

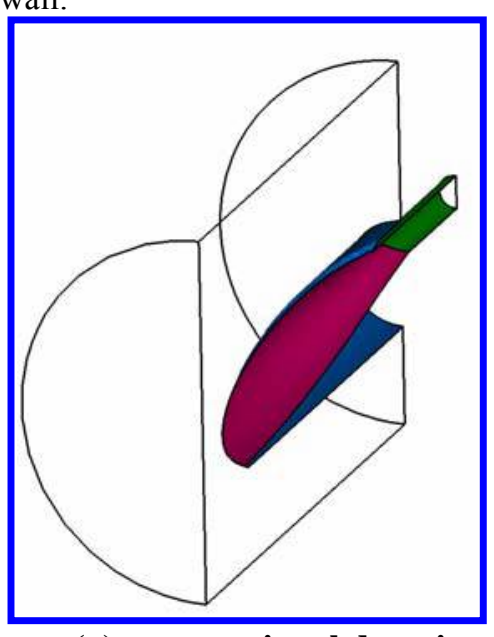

(a)computational domain

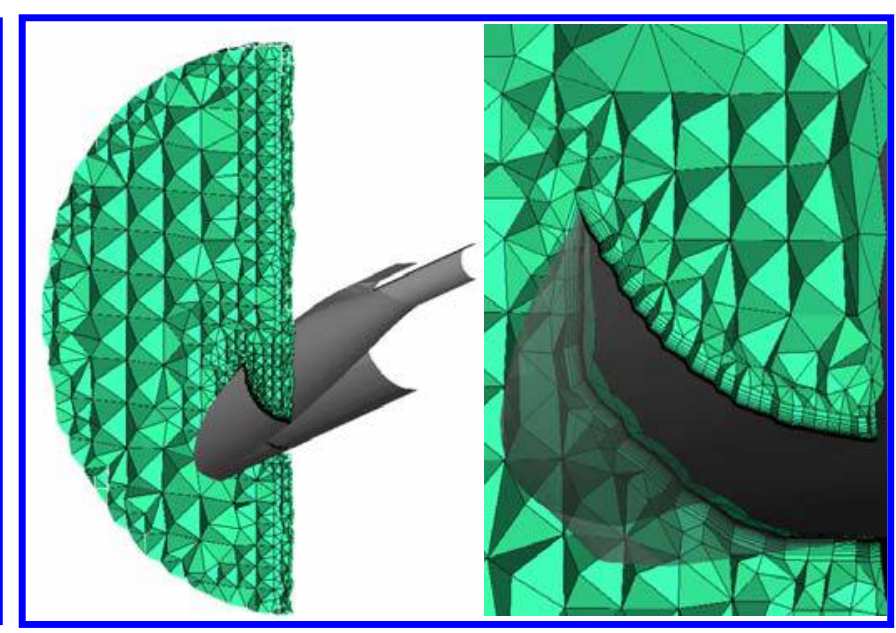

(b) computational grid

(c) computational grid enlarged 


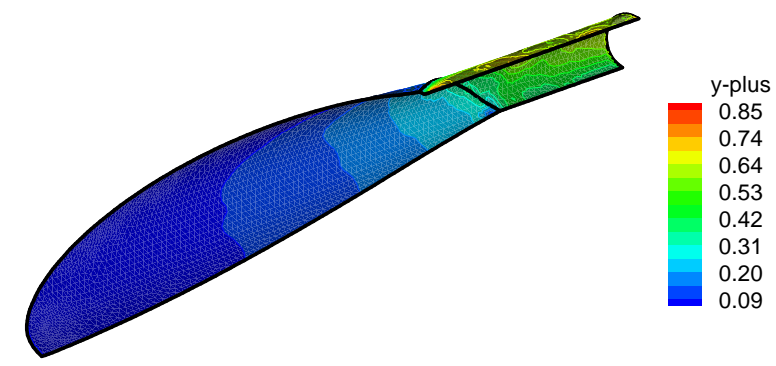

(d) wall $\mathbf{y}^{+}$number

Fig 11. The computational domain, computational grid and wall $\mathrm{y}^{+}$

Table 2: Description of all cases to analyze the inlets

\begin{tabular}{|c|c|c|c|c|c|}
\hline \multicolumn{2}{|c|}{ Case } & Inlet type & $\begin{array}{c}\text { Baseline flow field } \\
\text { Truncation }\end{array}$ & $\begin{array}{c}\text { Correcti } \\
\text { on }\end{array}$ & Viscosity \\
\hline \multirow{2}{*}{1} & A & 2D axisymmetric & None & Yes & Turbulent \\
\cline { 2 - 6 } & B & 2D axisymmetric & AOT $=5.5 \mathrm{deg}$ & Yes & Inviscid \\
\cline { 2 - 6 } & C & 2D axisymmetric & AOT $=5.5 \mathrm{deg}$ & Yes & Turbulent \\
\hline \multirow{2}{*}{2} & A & 3D stream-traced & AOT $=5.5 \mathrm{deg}$ & No & Inviscid \\
\cline { 2 - 6 } & B & 3D stream-traced & AOT $=5.5 \mathrm{deg}$ & No & Turbulent \\
\hline
\end{tabular}

The free-stream conditions chosen are Mach 5.8, 3000Pa static pressure and a fixed temperature at $255 \mathrm{~K}$. Two different cases are examined for the 2D and 3D stream traced inlets. The first case is to examine the improvement of the viscous correction. The second case is to provide an understanding of the internal flow within the stream-traced inlets with AOT of 5.5deg, see table 2.

The correction effect and the mass flow weighted performance are investigated For two dimensional cases, the $\mathrm{x}$ coordinate stands for axial distance from the original point and y coordinate stands for radial distance from the symmetric axis. The dimension in all 2D and 3D cases is meter.

\section{Results of two dimensional models}

The flow pattern for Case1A is shown in figure 12. After correction, the Mach contours coalesce at the origin point, the core flow is restored to the designed pattern ( compared with figure 5a). The total pressure recovery of the core flow has been improved up to $90 \%$ (see figure $12 \mathrm{c}$ ). The correction is successsful in restoring the core flow pattern to its design.
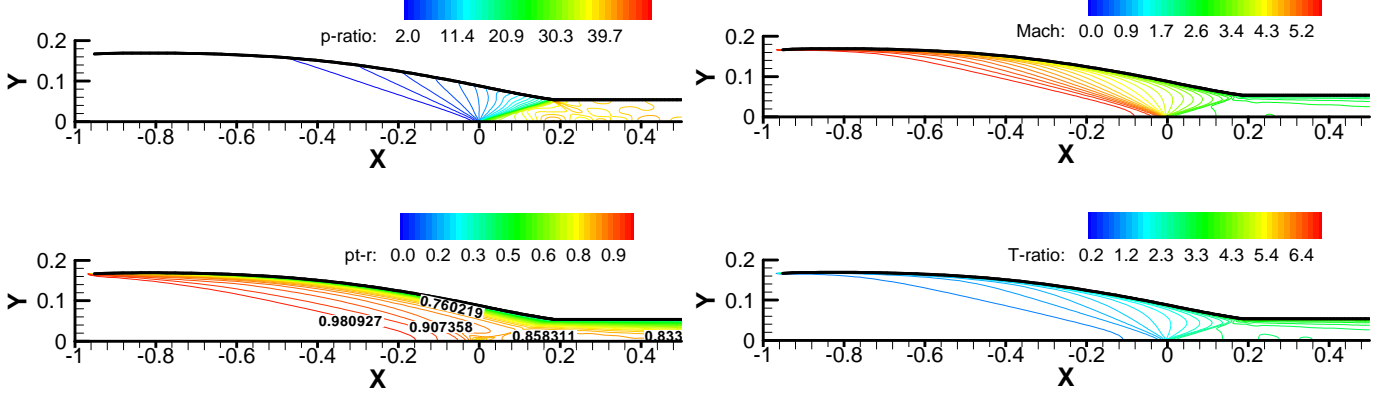

Fig 12. Contours of $2 D$ axisymmetric corrected full Busemann inlet 
The comparison of flow patterns for Case $1 \mathrm{~B}$ and $1 \mathrm{C}$ are shown in figure 13 and 14. For the case of 1B, the coalescence of Mach waves at the origin point is not realized. The shockwave emanated from leading edge is internally curved and intersects with the symmetric axis at $20 \mathrm{~mm}$ behind the origin, see figure 13. The reflected conical shock wave intersects with the wall of isolator $3 \sim 7 \mathrm{~mm}$ behind the shoulder. This will lead to a small re-expansion region, and there will be a with a pressure trough (due to the expansion) followed by an immediate pressure peak (due to the shock) on the wall behind the shoulder, see figure 15 .

After correction, the Mach number of the core flow and pressure ratio contours of the entire flow of the corrected inlet agree well with those of the inviscid one, see figure 14.

The total pressure recovery of the core flow has been improved up to $90 \%$ (see figure $12 \mathrm{c}$ ). The correction is successful in restoring the flow pattern to its design. The wall pressure shows excellent agreement between the inviscid inlet and the viscid corrected inlet. The sudden pressure rises that represents reflected shock waves agree well. The correction is successful for Case 1.

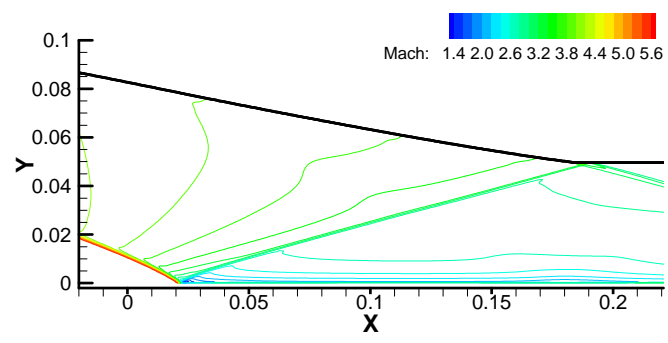

(a)

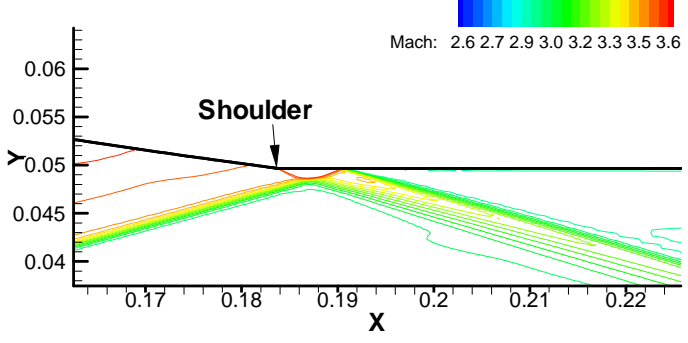

(b)

Fig 13. shock structure of Case 1B

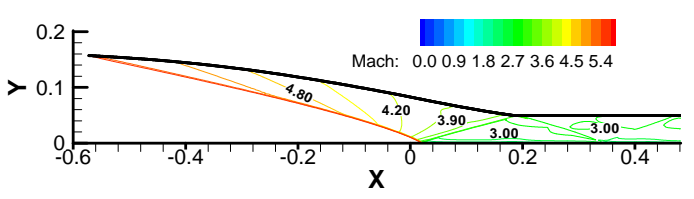

(a) Case 1B, Mach number

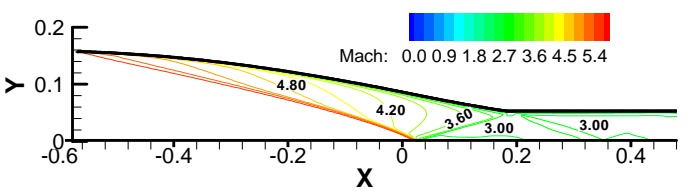

(c) Case 1C, Mach number

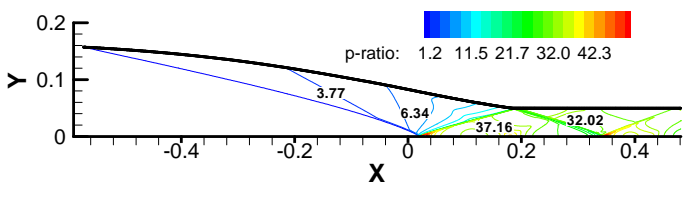

(b) Case 1B, pressure ratio

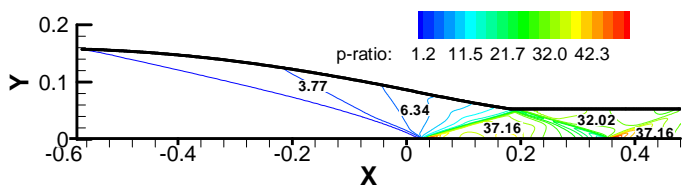

(d) Case 1C, pressure ratio

Fig 14. Flow pattern of Case $1 B$ and $1 C$

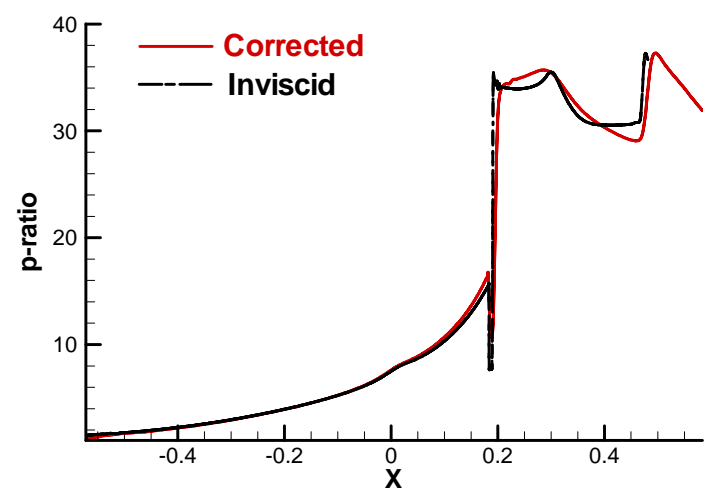


Fig 15. Wall pressure of inviscid and corrected inlet

\section{Results of three dimensional models}

The model to be computed is CCS-TBI-5.5, which has a total contraction ratio of 7.9 and an internal con- traction ratio of 2.6 (see fig6c).

For the inviscid and corrected cases, the flow patterns are shown in figure 16. For the inviscid case, the distribution of the iso-surfaces of pressure ratio is as designed. As mentioned above, the turbulent computational result reports the unstart (see figure 8) of the uncorrected stream-traced inlet CCS-TBI-5.5 (figure 6c). Whereas, the boundary layer correction has restored the pressure distribution to the invicid pattern(figure 16b). Wall pressure of two lines are quantitatively compared between the invicid and corrected inlet. Line 1 is the intersection between the symmetry plane and the bottom side of the wall. Line 2 is the intersection between the wall and the plane that $\mathrm{y}=0.025 \mathrm{~m}$, which divides the isolator equally ( $\mathrm{s}$ ee figure 17).

Figure $17 \mathrm{~b}$ shows that, the pressure on line 1 of the corrected inlet and the inviscid inlet agree well before the point $\mathrm{x}=0.16$ ( $\mathrm{x}=0.183$ is the start point of the isolator), the reflected shock wave impinges at the wall of the corrected inlet is $20 \mathrm{~mm}$ before that of the inviscid inlet. The pressure on line 2 of the corrected inlet and the inviscid inlet agree well before the point $\mathrm{x}=0.092$, the reflected shock wave impinges at the wall of the corrected inlet is $30 \mathrm{~mm}$ before that of the inviscid inlet. From the the correction is effective on the external compression stage, but not as excellent near the throat. From figure 17, we can see the compression manner of the internal wave rider inlet, and there is not any leakage of mass flow from the high pressure region to the ambient pressure region. The flow in the isolator is simple and relatively uniform, as shown in the figure 18 . The mass flow is $100 \%$ captured and the mass weighted total pressure recovery is $51.8 \%$ at the exit of the isolator whose length is $400 \mathrm{~mm}$.

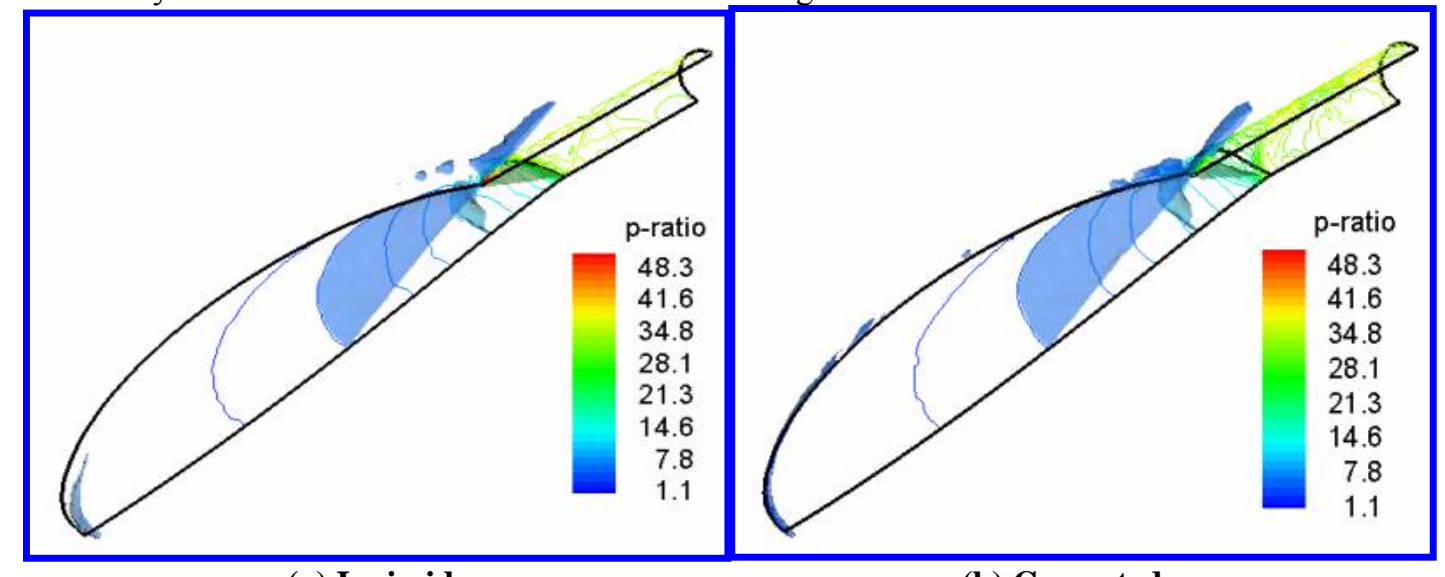

(a) Inviscid

(b) Corrected

Fig 16. ISO-surfaces and contours of the pressure ratio 


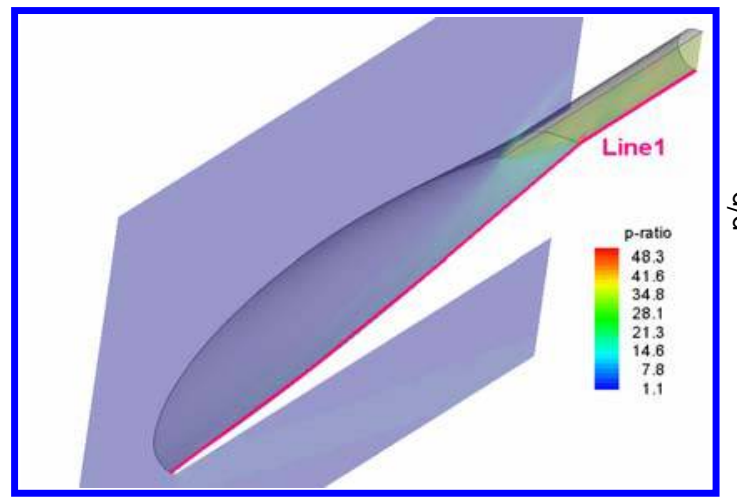

(a) Line1: intersection of the symmetry plane and the bottom side of the wall

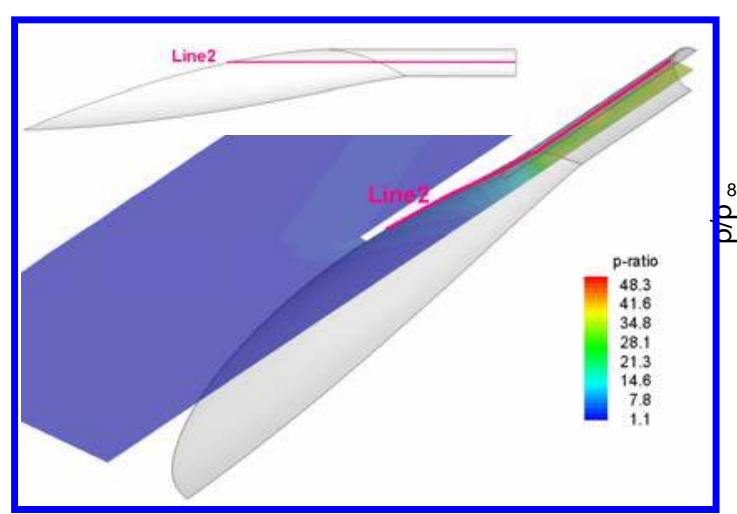

(c) Line1: intersection of the symmetry plane and the bottom side of the wall

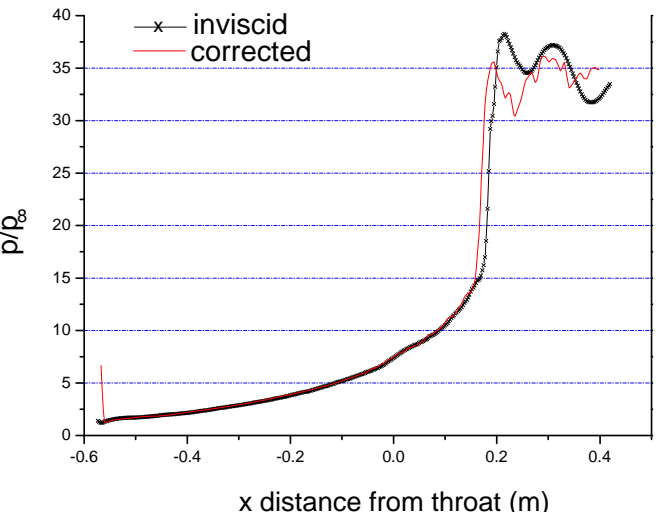

(b) Wall pressure on the line 1 of the corrected inlet vs that of the invicid inlet

Fig 17. Wall pressure comparison between the inviscid and the corrected inlet on line1 and line2

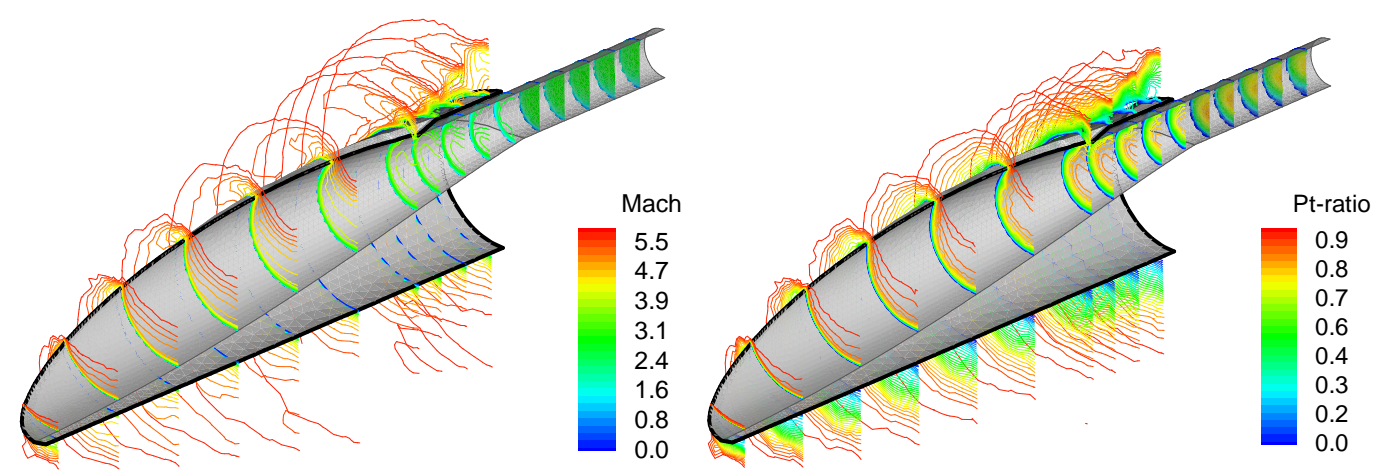

Fig 18. Mach number and total pressure ratio contours within the corrected CCS-TBi-5.5 inlet 

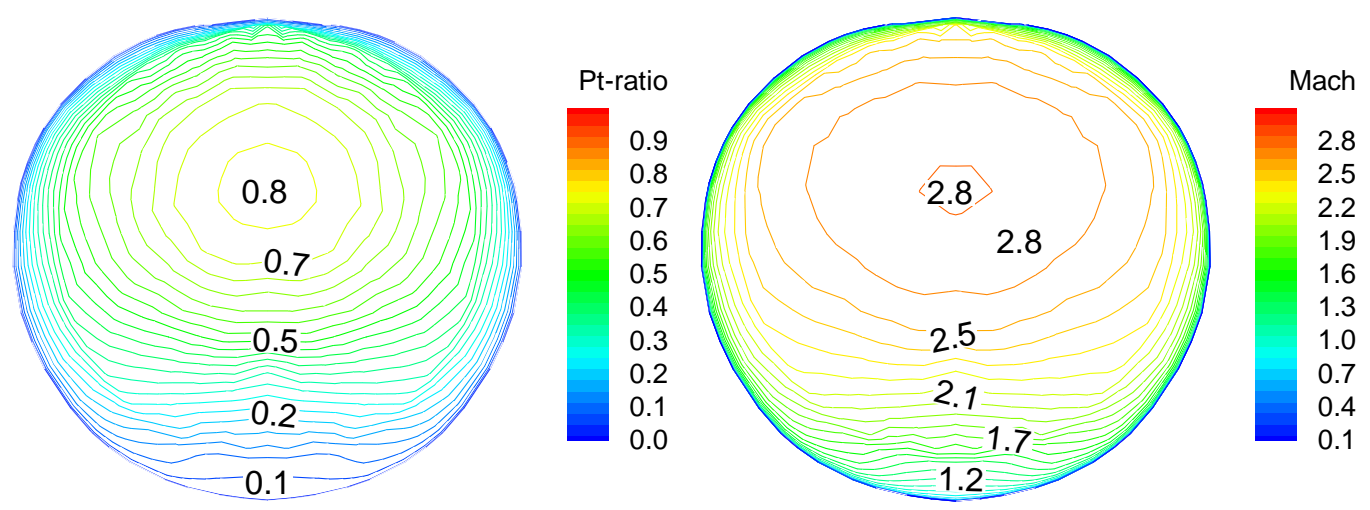

Fig 19. Mach number and total pressure ratio contours at the exit of the isolator of the corrected CCS-TBi-5.5 inlet

\section{Conclusions}

In this paper, a type of stream-traced inward turning inlet is designed based on the truncated Busemann flowfield. Boundary layer correction is applied to the 2D axisymmetric and 3D stream traced inlets, to restore the flow field to their designs. CFD tools has been used to analyze the internal flow pattern. The correction on the 2D axisymmetric and is successful in restoring the core flow pattern to its design. For 3D cases, the correction is successful for the external compression stage, but the reflected shock is $20-30 \mathrm{~mm}$ before its position of the inviscid case. The flow in the isolator is simple and relatively uniform. The incoming mass flow is $100 \%$ captured and the mass total pressure recovery is up to $52 \%$ at the exit. The inlet shows a good performance.

\section{Acknowledgment}

The authors acknowledge the valuable help of Dr. Bo Sun of Nanjing University of Aeronautics and Astronautics, on the topics covered in this paper.

\section{References}

[1] Mölder, S.Internal, axisymetrric, conical flow [J].AIAA Journal,1967,5(7).

[2] Billig,F.S., Design and Analysis of Streamline Traced Hypersonic Inlets, AIAA 99-4974.

[3] Billig,F.S., Streamline Tracing: Technique for Designing Hypersonic Vehicles, Journal of Propulsion and Power, Vol.16, No.3, May-June-2000.

[4] Matthews,A.J., Jones,T.V., Design and Test of a Modular Waverider Hypersonic Intake, Journal of Propulsion and Power, Vol.22, No.4, July-August-2006.

[5] Walsh, P.C., Molder,S., Boundary layer Correction for the Busemann Hypersonic Air Inlet, Can. Aeronautics and Space J., Vol. 49, No. 1, pp. 11-17, 2003.

[6] Ceberci,Tuncer., Bradshaw.Peter., Physical and computational aspects of convective heat transfer, Springer-Verlag New York Inc, 1984. 